Conclusion Thrombolysis rates in New Zealand continue to rise and now surpass the more recent $10 \%$ Ministry of Health target. The continued reduction in door-to-needle time is also an indication of continued service improvement resulting in better patient outcome. However, there are still to be opportunities for improvement.

\section{CASE REPORT: TO STENT, OR NOT TO STENT? THE PALE CAST OF EVIDENCE-BASED PRACTICE IN ACUTE STROKE}

${ }^{1,2}$ Chris Blair*, ${ }^{3,4}$ Kartik Bhatia, ${ }^{4}$ David Brunacci, ${ }^{2}$ John Worthington, ${ }^{2}$ Rebekah Ahmed. ${ }^{1}$ Department of Neurology, Liverpool Hospital, Sydney, NSW, Australia; ${ }^{2}$ Department of Neurology, Royal Prince Alfred Hospital, Sydney, NSW, Australia; ${ }^{3}$ Department of Interventional Neuroradiology, Toronto Western Hospital, Toronto, Ontario, Canada; ${ }^{4}$ Department of Interventional Neuroradiology, Royal Prince Alfred Hospital, Sydney, NSW, Australia

\subsection{6/jnnp-2019-anzan.89}

Introduction With approximately 200 procedures performed in the last year at our centre, worthwhile clinical lessons continue to emerge in the practice of endovascular clot retrieval (ECR) for acute stroke. This case demonstrates the value of considered clinical appraisal in a dynamic, information-rich setting. A 68 year-old man with established vertebrobasilar atherosclerotic disease developed capricious, blood pressuresensitive neurological deficits after successful ECR for a basilar artery stroke, inviting the possibility of further intervention in the form of intracranial stenting. We avoided pursuing this course of action in favour of a more measured approach, entailing the provision of vasopressor support over the following week.

Method Following ECR, our patient was admitted to the intensive care unit for continuous blood pressure monitoring and close observation of his neurological deficits with serial NIHSS (National Institutes of Health Stroke Scale) scoring. Systolic blood pressures were maintained between 140-160 $\mathrm{mmHg}$ using vasopressor support, with the aim of allowing time for recovery of vascular autoregulation and collateralization.

Results Over six days, the patient developed moderate left upper and lower limb weakness. An MRI performed on Day 5 revealed limited interval infarction of the right hemipons and cerebellum, with complete re-occlusion of the mid-basilar arterial segment. He left the ICU with a NIHSS score of 7 , and was living independently at 90-day follow-up (Modified Rankin Score 1).

Conclusion The ultimately favourable net outcome for our patient clearly illustrates the imperative to remain within the boundaries of evidence-based practice in this bold and rapidly evolving discipline.

\section{LATE-ONSET PARADOXICAL REACTIONS IN NEUROTUBERCULOSIS PRESENTING 18 MONTHS AND 20 YEARS AFTER TREATMENT}

${ }^{1}$ Ruth Leadbetter*, ${ }^{2}$ Timothy Blackmore, ${ }^{1}$ lan Rosemergy. ${ }^{1}$ Neurology Department, Wellington Hospital, Wellington, New Zealand; ${ }^{2}$ Infectious Diseases Department, Wellington Hospital, Wellington, New Zealand

10.1136/jnnp-2019-anzan.90

Introduction Paradoxical reactions (PRs) during treatment of neurotuberculosis are common but late-onset PRs after completion of anti-tuberculous treatment are reported very rarely. The management of late-onset PRs is challenging as excluding other diagnoses is difficult without invasive testing.

Methods Two cases of late-onset PRs after treatment for neurotuberculosis are reported. The timing of onset, clinical and radiological features of these PRs are described and the diagnostic work up and outcomes after empiric immunosuppression reviewed.

Results A 24-year-old woman presented with right-sided focal motor seizures 18 months after receiving anti-tuberculous treatment for neurotuberculosis. MRI showed enlargement of a previous left frontal tuberculoma with extensive oedema. A biopsy was not performed as the lesion involved eloquent brain and the patient declined a lumbar puncture. She was treated empirically with corticosteroids for six months and had clinical and radiological improvement.

A 56-year-old woman presented with left leg weakness and numbness 20 years after treatment for multiple cerebral tuberculomas. MRI showed a new confluent area of T2 hyperintensity in the right frontal and parietal lobes. Cerebrospinal fluid was negative for acid-fast bacilli. She was treated with corticosteroids for 2 months with progressive improvement.

Conclusions Late onset PRs to neurotuberculosis can occur months to years after completing anti-tuberculous therapy. Recognising this entity is important so unnecessary treatments and brain biopsy may be avoided. Empiric immunosuppression with corticosteroids appears to be safe if early follow up and repeat imaging is arranged.

\section{NEW ZEALAND HOSPITAL STROKE SERVICE PROVISION: A NATIONAL SURVEY}

'Stephanie Thompson, ${ }^{2} J e r o e n$ Douwes, ${ }^{3}$ Alan Barber, ${ }^{4}$ Dominique Cadilhac, ${ }^{5}$ Harry McNaughton, ${ }^{6} J o h n$ Gommans, ${ }^{7}$ John Fink, ${ }^{8}$ Alan Davis, ${ }^{9}$ Valery Feigin, ${ }^{1}$ Anna Ranta*. 'Capital and Coast District Health Board and University of Otago, Wellington, Wellington, New Zealand; ${ }^{2}$ Massey University, Wellington, New Zealand; ${ }^{3}$ University of Auckland, Auckland, New Zealand; ${ }^{4}$ Monash University, Melbourne, VIC, Australia; ${ }^{5}$ Medical Research Institute of New Zealand, Wellington, New Zealand; ${ }^{6}$ Hawke's Bay Hospital, Hastings, New Zealand; ${ }^{7}$ Christchurch Hospital, Christchurch, New Zealand; ${ }^{8}$ Northland DHB, Whangarei, New Zealand; ${ }^{9}$ Auckland University of Technology, Auckland, New Zealand

\subsection{6/jnnp-2019-anzan.91}

Introduction The REGIONS Care study assesses stroke care throughout New Zealand. It includes a national audit and here we present organisational survey results assessing current availability of interventions and management approaches in each hospital.

Methods All 20 District Health Boards were invited to complete a survey about patients managed per annum, care setting, and service provision. Results were grouped into rural and urban groups to assess for geographic differences.

Results All NZ hospitals managing stroke patients completed the survey. Results found that of these hospitals 92\% have an acute stroke unit, $85 \%$ have a TIA pathway, but only $58 \%$ offer rapid access specialist TIA services. 100\% offer thrombolysis, 92\% use pre-hospital notification and rapid ED triage system, and 73\% an in-hospital 'code stroke' alert. 50\% have access to thrombectomy. Only $32 \%$ offer a dedicated inpatient stroke rehabilitation unit. While $92 \%$ provide community rehabilitation, only $11 \%$ offer early supported discharge programmes. $87 \%$ routinely provide stroke patient education, but only $54 \%$ provide individualised stroke care plans at 
discharge. Only $28 \%$ have access to a psychologist. In-hospital stroke alerts $(p=0.014)$, access to thrombectomy $(p=0.016)$, access to stroke unit care $(\mathrm{p}=0.027)$, and routine referral to stroke foundation $(p=0.049)$ were more common in urban areas.

Conclusion The results of this organisational survey indicates that stroke care provision has improved since the last audit in 2009, but important gaps remain. These results will help services focus on specific areas for improvement, some of which such as pre-hospital alerts should be relatively easy to address.

\section{SILENT MULTILEVEL VERTEBRAL FRACTURES IN A SEVERE CASE OF GLYCINE RECEPTOR ANTIBODY- POSITIVE PROGRESSIVE ENCEPHALOMYELITIS WITH RIGIDITY AND MYOCLONUS (PERM)}

Myintzu Min*, Han Liu*, Paul Silberstein. Royal North Shore Hospital, Sydney, NSW, Australia

\subsection{6/jnnp-2019-anzan.92}

Introduction Autoimmune encephalitides are a heterogenous and potentially devastating group of disorders. Antibodies to glycine receptor (GlyR) are rare and increasingly reported in patients with progressive encephalomyelitis with rigidity and myoclonus (PERM). PERM patients develop early brainstem and autonomic dysfunction, and if untreated, can be fatal. We aim to discuss the complications and treatments in this PERM case and review the literature on management of PERM.

Methods We report a case of PERM currently undergoing treatment in our hospital.

Results Mr GT is a 46-year-old male who presented with a prodromal phase of altered sensation and acute cerebellar signs. He rapidly deteriorated with bulbar dysfunction and developed generalised muscle rigidity and hyperreflexia. Infective work-up, cerebrospinal fluid analysis, magnetic resonance imaging of brain and spine were initially normal. He was started on first line immunosuppressants for a clinical diagnosis of autoimmune encephalitis. Despite sedation in intensive care, generalised myoclonus and truncal extensor spasms were severe and presented on-going management difficulties. A computed tomography scan of his abdomen to exclude malignancy revealed incidental findings of vertebral fractures at multiple thoracic and lumbar levels. $\mathrm{He}$ was subsequently administered rituximab and demonstrated some signs of recovery. GlyR antibody positive results from Oxford University Hospital consequently confirmed his diagnosis.

Conclusion This is the fourth reported GlyR antibody-positive PERM case in Australia and the first description to our knowledge with intractable rigidity and myoclonus leading to vertebral fractures. Awareness and screening for this complication may assist in management of patients with PERM.

\section{A PRESENTATION RESEMBLING TRANSIENT GLOBAL AMNESIA WITH UNDERLYING TAKO-TSUBO CARDIOMYOPATHY AND MULTIFOCAL ISCHAEMIC STROKE (WITH RESTRICTED DIFFUSION IN THE LEFT MESIOTEMPORAL LOBE)}

Kristen Lefever, Chloe Tyson, Lisa Shelley, Daniel Schweitzer, Andrew Swayne*. Centre for Neurosciences, Mater Hospital, Brisbane, QLD, Australia

10.1136/jnnp-2019-anzan.93
Introduction We present a case of a 65 year-old female with a presentation resembling transient global amnesia (TGA) with investigations revealing a Tako-tsubo cardiomoyopathy and multifocal punctate areas of restricted diffusion consistent with ischaemic stroke.

Methods Single case report.

Results A 65 year-old female presented altered behaviour with anterograde amnesia consistent with an episode of TGA. There was no clearly identifiable stressor to provoke this episode. The TGA-like symptoms persistent for 12 hours before the patient made a full recovery.

During the presentation the patient complained of chest pain with elevated troponin. Cardiac investigation was notable for mild left ventricular dilation with mid- and apical hypokinesis on transthoracic echocardiogram and a normal coronary angiogram consistent with Tako-tsubo cardiomyopathy. Magnetic resonance imaging of the brain revealed multifocal infarcts including punctate foci of restricted diffusion within the left mesiotemporal lobe.

Conclusion This case is notable as it is rare to have a combined presentation with TGA-type symptoms with Tako-tsubo cardiomyopathy and multifocal ischaemia stroke. ${ }^{1}$ This is the first clinical case presentation involving the aforementioned triad and mesiotemporal lobe restricted diffusion. We hypothesise that the Tako-tsubo cardiomyopathy acted as the triggering event and the mesiotemporal lobe involvement caused the TGA-like symptoms thereby accounting for the clinical presentation and investigation findings. As mesiotemporal/hippocampal restricted diffusion may be seen with transient global amnesia, ${ }^{2}$ this case is of special interest mechanistically although we cannot exclude the alternative hypothesis that the episode of TGA resulted in the Takotsubo cardiomyopathy which subsequently caused the cardioembolic shower.

\section{REFERENCES}

1. Stollberger C, DeCillia N, Finsterer J. 'Tako-tsubo cardiomyopathy with transient global amnesia and cerebellar embolic stroke triggered by existential fear.' Neurol Neurochir Pol 2018; 52(3):394-396.

2. Enzinger C, Thimary F, Kapeller P, Ropele S, Schmidt R, Ebner F, Fazekas F. 'Transient global amnesia: diffusion-weighted imaging lesions and cerebrovascular disease.' Stroke 2008:39(8):2219-2225.

\section{HAEMORRHAGIC SPINAL CORD INFARCT - A RARE COMPLICATION OF SYMPATHOMIMETIC AMINE TOXICITY}

Ellen L Wall*, Jerome A Leow, Jonathan Ho, Yun T Hwang. Central Coast Local Health District, Gosford, NSW, Australia

10.1136/jnnp-2019-anzan.94

Introduction Sympathomimetic amines are recreational substances, available illegally as amphetamine derivatives (eg, 'ecstasy' and 'speed'). Ingestion can lead to significant medical complications such as hyperthermia, tachyarrhythmia, seizures and strokes, attributed to catecholamine surge and sympathetic overstimulation.

Method We report an unusual case of sympathomimetic amine ingestion manifesting as hypertension followed by acute onset flaccid paralysis of lower limbs bilaterally and T11 level anaesthesia in a 64 year old woman secondary to haemorrhagic cord infarct.

Results An MRI spine showed features compatible with spinal cord infarction from thoracic level $6 / 7$ to conus associated 